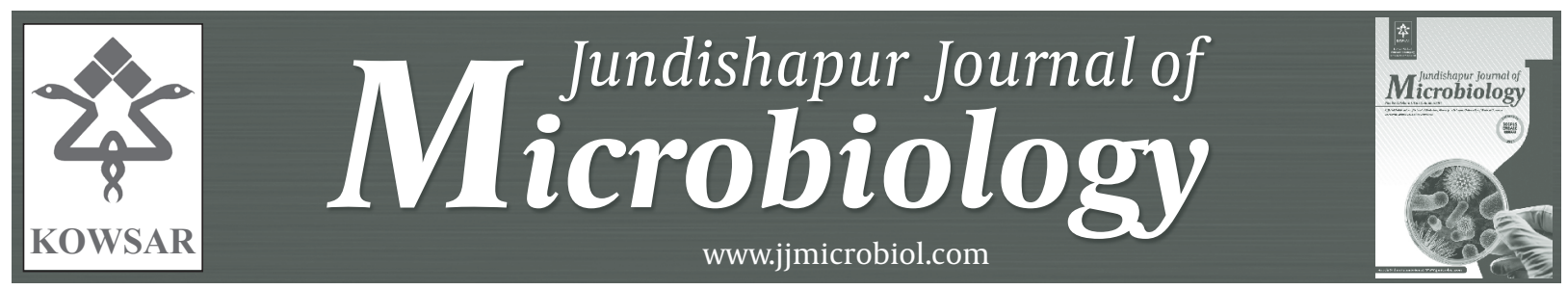

\title{
L-Tryptophan Production by Whole Cells of Escherichia coli Based on Iranian Sugar Beet Molasses
}

\author{
Elnaz Faghfuri ${ }^{1, *}$, Jamshid Fooladi ${ }^{1}$, Shayesteh sepehr ${ }^{1}$, Seyedeh Zahra Moosavi-Nejad ${ }^{1}$ \\ ${ }^{1}$ Department of Biology, Alzahra University, Tehran, IR Iran \\ ${ }^{*}$ Corresponding author: Elnaz Faghfuri, Department of Biology, Faculty of Science, Alzahra University, Tehran, IR Iran . Tel.: +98-9141052527, E-mail: elnaz. \\ faghfuri@gmail.com.
}

\begin{abstract}
A B S T R A C T
Background: L-tryptophan (L-Trp) is a nutritionally essential amino acid that the body uses to synthesize proteins, niacin (vitamin B3) and serotonin (neurotransmitters). Human and animals depend on plants and microorganisms for its supply. Remarkable increasing demand has caused the development of a wide variety of biotechnological methods for its production which work as well for other amino acids.

objectives: The present work reports on the use of Iranian sugar beet molasses as an inexpensive source of L-serine (precursor) and PLP (cofactor) for the production of L-Trp by Escherichia coli (ATCC 11303). Carbon source and buffered condition are also optimized for L-Trp production by E. coli.

Materials and Methods: E. coli (ATCC 11303) was used as the microbial source of tryptophan. Batch fermentation was performed with $8 \mathrm{~L}$ sugar beet molasses medium. The fermentation was carried out at $37^{\circ} \mathrm{C}$ with an agitation speed at $250 \mathrm{rpm}$. The pH was controlled at 7 with $10 \mathrm{~N}$ $\mathrm{NaOH}$. The cells were recovered by centrifugation. A $1 \mathrm{~g}$ mass of $E$. coli cells (wet mass) was used as the biocatalyst in the reaction medium (100 cc) containing (in grams/100cc): Molasses/glucose $=3.5$; Indole $=0.2 ; \mathrm{L}-\mathrm{Serine}=0.35 ; \mathrm{PLP}=0.005 ;(\mathrm{NH} 4) 2 \mathrm{SO} 4=0.5$. The reaction medium was incubated on rotatory shaker $(180 \mathrm{rpm})$ for $8 \mathrm{~h}$ at $37^{\circ} \mathrm{C}$. The reaction was carried out in $\mathrm{KH} 2 \mathrm{PO} 4-\mathrm{K} 2 \mathrm{HPO} 4$ buffering system $(\mathrm{pH}=8,0.1 \mathrm{M})$. The detection of produced L-Trp was carried out by the use of chromatography methods (HPLC and TLC), and fluorescence analysis.

Results: Consequently, the potassium phosphate buffer system was considered as the suitably buffered reaction medium. The concentration of L-Trp produced in reaction medium was $0.3 \mathrm{mM}$ and $0.12 \mathrm{mM}$ for glucose and molasses (a combination of sugar beet and cane molasses), respectively.

Conclusions: our present study shows that sugar beet molasses is a source of PLP and L-Ser; and hence, suggests the general application of the approach to produce L-Trp more economically.
\end{abstract}

Keywords: Escherichia coli; L- Tryptophan; Molasses

Copyright ( (2013, Ahvaz Jundishapur University of Medical Sciences; Published by Kowsar Corp.

Article type: Research Article; Received: 05 May 2012; Revised: 20 Jun 2012; Accepted: 03 Jul 2012; Epub: 01 Jun 2013; Ppub: Jun 2013

-Implication for health policy/practice/research/medical education:

L- tryptophan (L-Trp) is nutritionally essential amino acid that the body uses to synthesize proteins, niacin (vitamin B3) and serotonin (neurotransmitters). Human and animals depend on plants and microorganisms for its supply. Iranian sugar beet molasses can be used as a cheap source of L- ser (precursor) and PLP (cofactor) required for L-Trp production by Escherichia coli.

Please cite this paper as:

Faghfuri E, Fooladi J, Sepehr S, Moosavi-Nejad SZ. L- Tryptophan Production by Whole Cells of Escherichia coli Based on Iranian Sugar Beet Molasses. Jundishapur J Microbiol. 2013;6(4):e5370. DOI: 10.5812/jjm.5370.

Copyright ( 2013, Ahvaz Jundishapur University of Medical Sciences; Published by Kowsar Corp.

This is an Open Access article distributed under the terms of the Creative Commons Attribution License (http://creativecommons.org/licenses/by/3.0), which permits unrestricted use, distribution, and reproduction in any medium, provided the original work is properly cited. 


\section{Background}

Among all standard amino acids, L-tryptophan (L-Trp) is one of the least abundant and most expensive to produce (1). It is an aromatic amino acid with the unique indole side-chain that makes it a fundamental precursor to a number of neurotransmitters required for the regulation of appetite, sleep, mood and stress response (2). It is nutritionally essential for human and animals, which, in turn, depend on plants and microorganisms for its supply (3).

The bacterial tryptophan synthase (TSase) is a $\alpha 2 \beta 2$ - complex that catalyzes the synthesis of L- Trp from indole and L- Serine (L- Ser) (4). Tryptophanase (TPase) catalyzes the degradation of L-Trp to pyruvate, ammonia, and indole, allowing Escherichia coli and many other Gram-negative bacteria to utilize Trp as a source of carbon, nitrogen, and energy. TPase can also carry out the reverse reaction and synthesize L-Trp from indole and L-Ser (or L-cysteine) or from pyruvate and ammonia (5). TSase and TPase are known as PLP dependent enzymes.

There are three known types of methods for L-Trp production, i.e. fermentation methods, enzymatic methods, and chemical methods. Enzymatic methods use enzyme or whole cells of microorganisms with TPase or TSase activity. Some examples of this method are the use of Escherichia coli as a source of TPase (6) or TSase (7). An example of fermentation methods is overproduction of L-Trp by Corynebacterium glutamicum using glucose as the starting material (8).

Fermentative processes help to utilize the agro-industrial wastes such as molasses (9). Molasses is a product of the sugar industry based on both cane and sugar beet. The use of molasses is well-known in the fermentation industry. It is substrate for the manufacture of ethanol, yeast and other biochemical process (10).

\section{Objectives}

This study focuses on the use of Iranian sugar beet molasses as an inexpensive source of L-serine (precursor) and PLP (cofactor) for the production of L-Trp by E. coli (ATCC 11303). Carbon source and buffered condition are also optimized for L-Trp production by E. coli.

\section{Materials and Methods}

\subsection{Microorganism}

The test organism, a wild-type strain of E. coli (ATCC 11303), was obtained as a lyophilized culture from Dehghan et al. (11).

\subsection{Chemicals}

L-Trp, L-Ser, PLP, and indole were purchased from Merck.
Sugar beet molasses and cane molasses was provided by Iranian sugar factory.

\subsection{Media}

The stock cultures were maintained on agar slants that contained (gr/100cc): glucose, 2.0; yeast extract, 2.5; (NH4)2SO4, 0.05; agar, 1.5 at $\mathrm{pH} 7$ and Temperature of $37^{\circ} \mathrm{C}$ for $16 \mathrm{~h}$. The cells growing on the newly prepared slants were inoculated into the preculture1 medium that contained ( $\mathrm{gr} / 100 \mathrm{cc})$ : glucose, 2.0; yeast extract, 2.5; (NH4)2SO4, 0.05 and incubated at $37^{\circ} \mathrm{C}$ and $180 \mathrm{rpm}$ for 6 h.

A 7.5 $\mathrm{mL}$ aliquot of the preculture 1 was then transferred to a 2 L Erlenmeyer flask containing broth medium (preculture2) and was cultivated at $37^{\circ} \mathrm{C}$ and $180 \mathrm{rpm}$ for $6 \mathrm{~h}$ to provide inoculum for the fermentor. The constituents of preculture 2 medium and incubation condition were identical to preculture1 medium. Batch fermentation was performed with $8 \mathrm{~L}$ sugar beet molasses medium that contained ( $\mathrm{gr} / 100 \mathrm{cc})$ : sugar beet molasses, 2; yeast extract, 2.5; (NH4)2SO4, 0.05 in a 10L MS-F1 fermentor. The fermentation was carried out at $37^{\circ} \mathrm{C}$ with an agitation speed at $250 \mathrm{rpm}$. The $\mathrm{pH}$ was controlled at 7 with 10 $\mathrm{N} \mathrm{NaOH}$. The cells were recovered by centrifugation at 4 ${ }^{\circ} \mathrm{C}$ and $12000 \mathrm{rpm}$ for $20 \mathrm{~min}$ and subsequently washed twice with a $0.9 \%(\mathrm{w} / \mathrm{v}) \mathrm{NaCl}$ solution.

\subsection{Reaction Medium}

The $1 \mathrm{~g}$ of E. coli cells (wet mass) was used as biocatalyst at reaction medium (100 cc) containing (g/100cc): molasses/glucose, 3.5; Indole, 0.2; L- serine, 0.35; PLP, 0.005; (NH4)2SO4, 0.5. The reaction medium was incubated on rotatory shaker (180 rpm) for $8 \mathrm{~h}$ at $37^{\circ} \mathrm{C}$. The reaction was carried out in $\mathrm{KH} 2 \mathrm{PO} 4-\mathrm{K} 2 \mathrm{HPO} 4$ buffering system $(\mathrm{pH}=8$, $0.1 \mathrm{M})$

\subsection{Amino acid Analysis}

Qualitative analysis of amino acids was performed by Thin-Layer chromatography (TLC) applying a $60 \mu \mathrm{l}$ sample on silica gel 60 F254. The paper was irrigated in n-Butanol: acetic acid: water (65: 13: 22) solvent system. It was then dried and sprayed with $0.1 \%$ ninhydrin solution. Colored spots of amino acids appeared upon drying at $110{ }^{\circ} \mathrm{C}$ for $10 \mathrm{~min}$ and were identified by computing the Rf values.

Scanning of the TLC plates was performed under ultraviolet light $(280 \mathrm{~nm})$, and implemented for the determination of the fluorescent L-Trp spots (12).Fluorescence spectra were recorded using a spectrophotometer (Cary Eclipse) where, the conditions were maintained as:

Spectral range $(285-600 \mathrm{~nm})$, scan rate $=600 \mathrm{~nm} / \mathrm{min}$ PMT voltage $(\mathrm{V})$ at medium 
Average time $=0.100 \mathrm{~s}$

Ex-slit and Em-slit $=5 \mathrm{~nm}$

Total content of L-Trp in the culture fluid was determined by HPLC (Kontron, Berlin, Germany) using a C18 column $(250 \times 4.6 \mathrm{~mm}, 5 \mu \mathrm{m}$, Knauer) with the mobile phase described earlier (13).The flow rate was set at $1.0 \mathrm{ml} /$ min. The detection of the L-Trp was carried out by a spectrophotometer at $254 \mathrm{~nm}$.

\section{Results}

\subsection{Investigation of E. coli Growth}

The results indicated that while $E$. coli was growing in the fermentor after incubation for $6 \mathrm{~h}$, the bacteria were at the end of exponential phase (Figure 1).

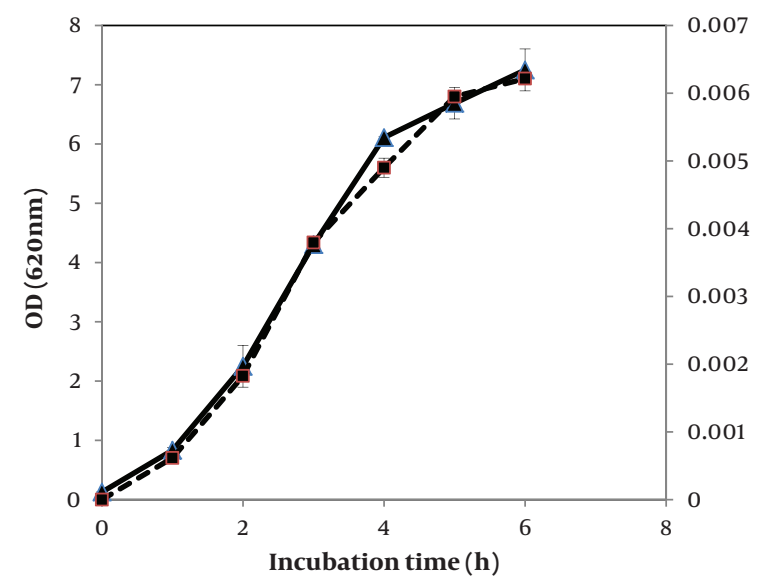

Figure 1. The growth curve of E. coli growing in the fermentor containing sugar beet molasses medium based on: the dry weight of biomass and the optical density of culture medium at $620 \mathrm{~nm}$. Temp $=37^{\circ} \mathrm{C}, \mathrm{pH}=$ $7, \mathrm{~N}=250 \mathrm{rpm}$.

At this time, the bacterial biomass was recovered and used as biocatalyst for tryptophan production.

\subsection{Effect of Different Buffers on L-Trp Production}

The results of this experiment indicate that the level of L-Trp production (Ctrp) in $\mathrm{KH} 2 \mathrm{PO} 4-\mathrm{K} 2 \mathrm{HPO} 4$ buffer (0.1 $\mathrm{M}, \mathrm{pH}=8)$ is higher than in other buffers $(0.1 \mathrm{M}, \mathrm{pH}=8)$. Other tested buffers included Tris-HCl, Borate-Borax, and Na2HPO4-NaH2PO4. The spectra of standard L-Trp spot on TLC plate revealed a major emission peak $(\lambda \mathrm{em})$ at $324 \mathrm{~nm}$ corresponding to an excitation wavelength ( $\lambda$ ex) of 280 $\mathrm{nm}$. The fluorescence intensity was studied at this wavelength (324 nm) for L-Trp spots, correlated to different reaction media based on different buffers ( Figure 2 ).

\subsection{Production of L- Tryptophan in Reaction Me- dium Containing Glucose}

The level of L-Trp production in reaction medium containing glucose was determined after $4 \mathrm{~h}$ of incubation.

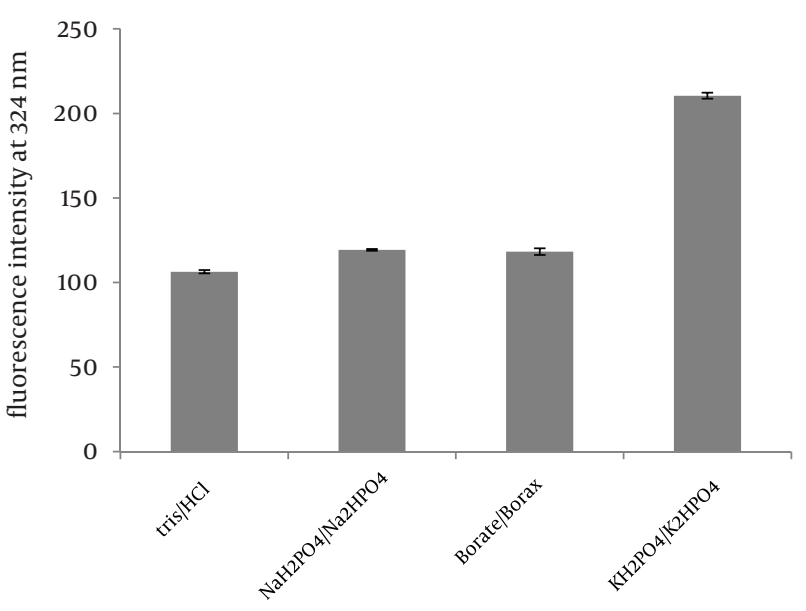

Figure 2. Fluorescence intensity of L-Trp spots on TLC plate correlated to different media based on different buffers. Temp $=37^{\circ} \mathrm{C}, \mathrm{pH}=8, \mathrm{~N}=$ $180 \mathrm{rpm}$
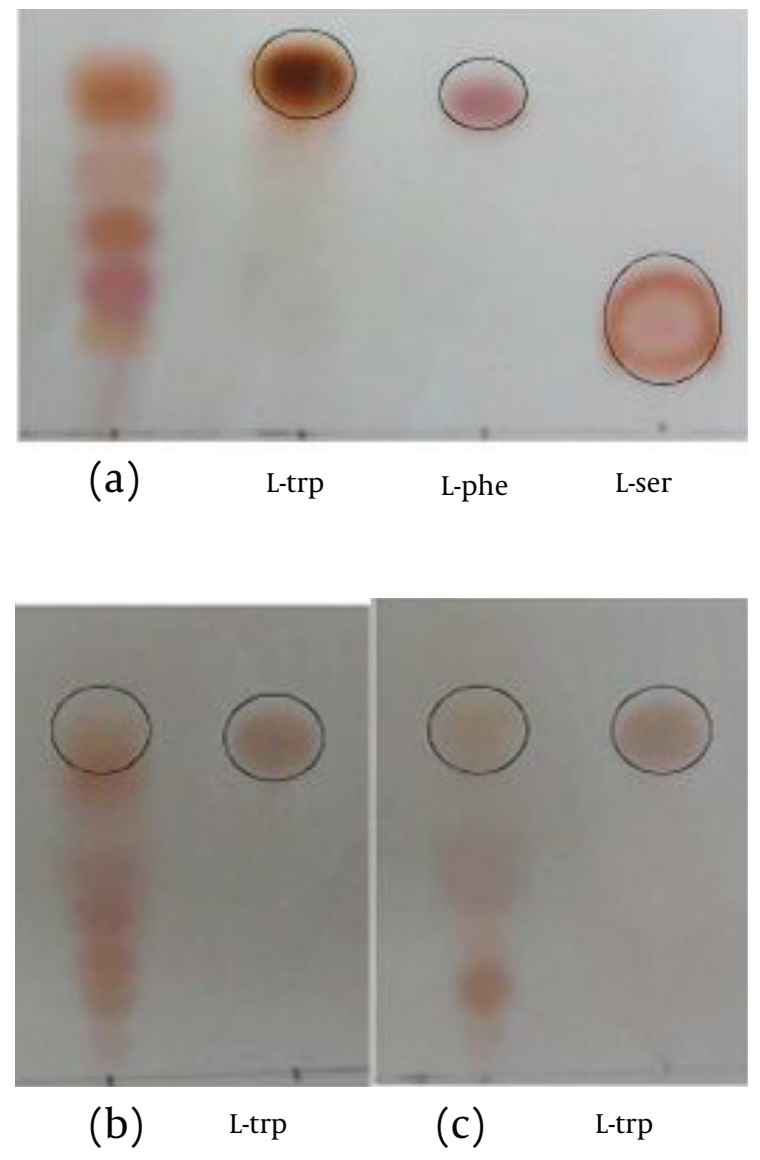

Figure 3. Qualitative analysis with TLC chromatogram of $60 \mu \mathrm{l}$ (a) organic phase of sugar beet molasses; and reaction medium containing: (b) sugar beet molasses; (c) cane molasses; in the presence of L-ser and PLP, with a solvent system of n-Butanol: acetic acid: water (65:13: 22).

According to HPLC analysis, Ctrp was increased to 0.3 $\mathrm{mM}$ in reaction medium after $4 \mathrm{~h}$. To examine the indole toxicity, bacterial biomass was incubated for $1 \mathrm{~h}$ with indole. In the reaction medium and in the presence of this 
biomass as biocatalyst, the level of L-Trp production was lower than in the previous experiment.

\subsection{Production of L-tryptophan in Reaction Medi- um Containing Sugar Beet and Cane Molasses}

TLC assay showed that the serine and phenylalanine constituted of the total free amino acid content of sugar beet molasses ( Figure $3 a$ ). Our results indicated that L-Trp production occurred in the reaction medium containing sugar beet molasses in the presence of L-Ser (precursor) and PLP (cofactor) ( Figure $3 b$ ). L-Trp production was observed when the reaction medium did not contain L-Ser and PLP.

When we used the reaction medium containing cane molasses, L-Trp production was observed in the presence of L-ser and PLP ( Figure 3c ). In the absence of L-Ser and PLP, L-Trp production was not observed.

Figure 4. Chromatogram Analysis: (1) L-Trp production in the reaction medium containing cane and sugar beet molasses combination; (2) standard L-Trp calibration curve.
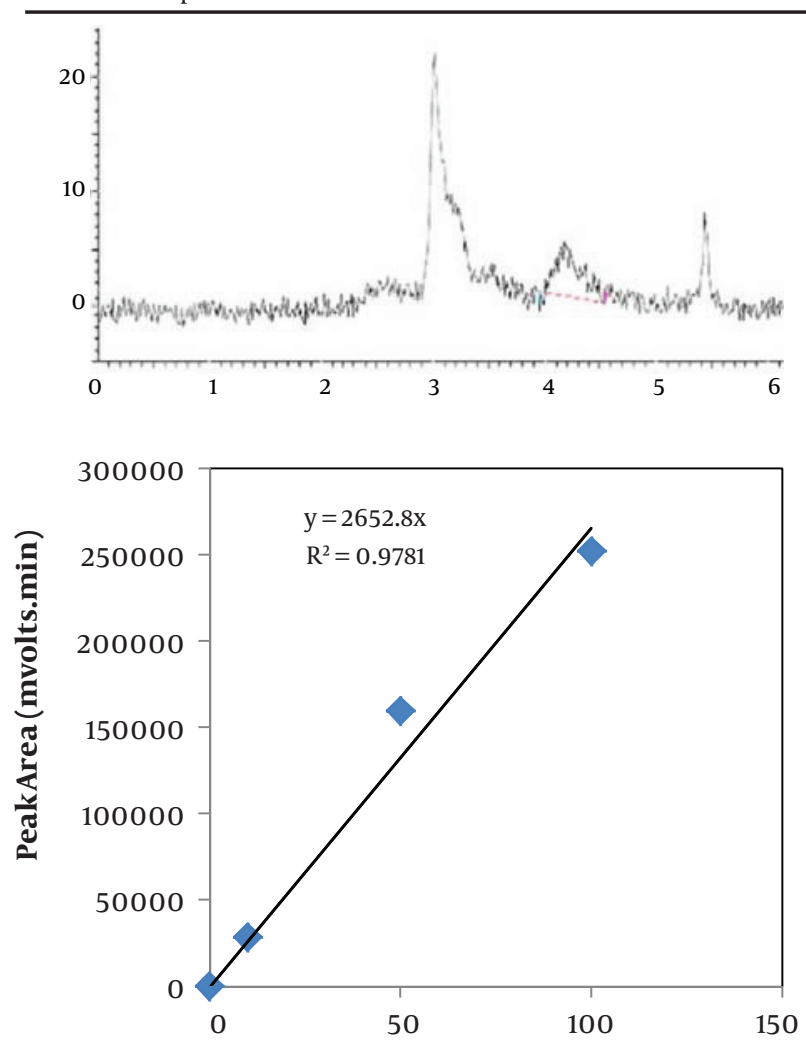

Concentration of L- Trp (ppm)

Other conditions: C18 column $(250 \times 4.6 \mathrm{~mm}, 5 \mu \mathrm{m})$; injection volume: $20 \mu \mathrm{l}$; UV detection at $254 \mathrm{~nm}$; retention time: $4.267 \mathrm{~min}$.

HPLC was used for the quantitative analysis of L-Trp production in the reaction medium. A linear relationship between Ctrp and the Peak-Area (mVolts $\times$ min) was observed over the concentration range (0-150 $\mathrm{ppm} / 20$ $\mu \mathrm{l})$. Chromatogram analysis showed the Peak-Area of 63805 (mVolts $\times$ min) in the reaction medium containing cane and sugar beet molasses. When we used both sugar beet and cane molasses in a reaction medium, L-Trp production (0.12 mM) was tangibly increased ( Figure 4 ).

\section{Discussion}

As the precursors of L-tryptophan, indole and L-serine have been used for the production of this amino acid (6, 7). The disadvantage of this method is the high cost of the precursors (14). As described earlier, Iranian sugar beet molasses can be used as a cheap source of L-Ser (precursor) and PLP (cofactor) required for L-Trp production. E. coli biomass obtained from fermentor containing sugar beet molasses medium can be used as a biocatalyst in the reaction medium in the absence of PLP or L-serine.

Our data suggest the integration of PLP into the cells growing on the sugar beet molasses. When we used both sugar beet and cane molasses in a reaction medium, LTrp production $(0.12 \mathrm{mM})$ was tangibly increased. The present results have revealed that L-Trp production was decreased when the whole cells was incubated for $1 \mathrm{~h}$ with indole. This might be due to the accumulation of indole, and/or a subsequent loss of cell viability. In a previous study (11), Dehghan and colleagues reported on the production of tryptophan by E. coli using Iranian cane molasses. Their data showed that Iranian cane molasses contains adequate amounts of both indole and serine enough for L-Trp production (0.48 mM).

In this study, TLC analysis showed the presence of Lserine and L-phenylalanine in the organic phase of sugar beet molasses. The conditions for production of L-tryptophan using whole cells of E. coli were optimized for carbon source and buffered condition. Potassium phosphate buffer was selected as the optimum to produce L-Trp. The influence of carbon source was investigated by replacing the molasses with glucose in the reaction medium. The higher L-Trp concentration was obtained as Ctrp = $0.3 \mathrm{mM}$ compared to that obtained with molasses. This result is compatible with the studies of Kocabash and colleagues on the fermentation characteristics of L-Trp production by Bacillus acidocaldarius (15). Their research indicated that carbon sources influence the operation of the intracellular network, and consequently the product formation.

Attempts to produce L-tryptophan during the growth of $E$. coli did not succeed, since the low concentrations of indole inhibited the growth of this organism. An analysis of the L-tryptophan metabolic pathway in another study (16) demonstrated that it is primary metabolite and its maximum production occurs in the logarithmic phase. The enzymes of tryptophan pathway are repressed during the growth in the presence of L-tryptophan. To avoid these effects, we have used the whole cells of E. coli. When precursors are employed, the complicated mutations of microorganism, which control the regulatory mecha- 
nism, are not required.

This study describes a cost-effective method for the identification of L-Trp. Thin-Layer Chromatography combined with fluorometric assay provides a simple and rapid technique for the estimation of free L-Trp concentration in the reaction medium.

In conclusion, our present study shows that sugar beet molasses is a source of PLP and L-Ser; and hence, suggests the general application of the approach to produce LTrp more economically. Further research focused on the quantitative analysis of the components of Iranian molasses is required.

\section{Acknowledgements}

None declared.

\section{Authors' Contribution}

None declared.

\section{Financial Disclosure}

None declared.

\section{Funding/Support}

None declared.

\section{References}

1. Ikeda M, Katsumata R. Hyperproduction of tryptophan by Corynebacterium glutamicum with the modified pentose phosphate pathway. Appl Environ Microbiol. 1999;65(6):2497-502.

2. Le Floc'h Nathalie, Seve Bernard. Biological roles of tryptophan and its metabolism: Potential implications for pig feeding. Live- stock Science. 2007;112(1-2):23-32.

3. Radwanski ER, Last RL. Tryptophan biosynthesis and metabolism: biochemical and molecular genetics. Plant Cell. 1995;7(7):921-34.

4. Miles EW, Moriguchi M. Tryptophan synthase of Escherichia coli. Removal of pyridoxal 5'-phosphate and separation of the alpha and beta2 subunits. J Biol Chem. 1977;252(19):6594-9.

5. Konan KV, Yanofsky C. Rho-dependent transcription termination in the tna operon of Escherichia coli: roles of the boxA sequence and the rut site. J Bacteriol. 2000;182(14):3981-8.

6. Mateus DM, Alves SS, Da Fonseca MM. Kinetics of L-tryptophan production from indole and L-serine catalyzed by whole cells with tryptophanase activity. J Biosci Bioeng. 2004;97(5):289-93.

7. Bang WG, Lang S, Sahm H, Wagner F. Production L-tryptophan by Escherichia coli cells. Biotechnol Bioeng. 1983;25(4):999-1011.

8. Ikeda M, Nakanishi K, Kino K, Katsumata R. Fermentative production of tryptophan by a stable recombinant strain of Corynebacterium glutamicum with a modified serine-biosynthetic pathway. Biosci Biotechnol Biochem. 1994;58(4):674-8.

9. Khan SH, Rasool G, Nadeem S. Bioconversion of cane molasses into amino acids. PakJ Agric Sci. 2006;43:157-160.

10. Roehr M. Weinheim (Federal Republic of Germany) VCH Verlagsgesellschaft $\mathrm{mbH}, ; 1996$.

11. Dehghan Shasaltaneh M, Fooladi J, Moosavi-Nejad SZ. L-tryptophan production by Escherichia coli in the presence of Iranian cane molasses. JPS. 2010;1(2):19-25

12. Gibbs CC, Saunders SJ, Sweeney GD. Quantitative estimation of tryptophan in plasma and urine using thin-layer chromatography (TLC) and induced fluorescence. Clin Chim Acta. 1967;17(3):31723.

13. Alegria A, Barbera R, Farre R, Ferreres M, Lagarda MJ, Lopez JC. Isocratic high-performance liquid chromatographic determination of tryptophan in infant formulas. J Chromatogr A. 1996;721(1):83-8.

14. Bang WG, Behrendt U, Lang S, Wagner F. Continuous production of L-tryptophan from indole and L-serine by immobilized Escherichia coli cells. Biotechnol Bioeng. 1983;25(4):1013-25.

15. Kocabaș P, Çalık P, Özdamar TH. Fermentation characteristics of l-tryptophan production by thermoacidophilic Bacillus acidocaldarius in a defined medium. Enzyme Microb Tech. 2006;39(5):1077-1088.

16. Ito J, Crawford IP. Regulation of the enzymes of the tryptophan pathway in Escherichia coli. Genetics. 1965;52(6):1303-16. 